\title{
Various Extensions of Original Born-Kramers-Slater Model for Reactions Kinetics Based on Brownian Motion and Fokker-Plank Equation Including 1D, 2D, 3D, and Multi-dimensional Approaches
}

\author{
Michael Fundator \\ DBASSE (Division of Behavioral and Social Sciences and Education) of the National Academy of Sciences, Engineering, and \\ Medicine, USA
}

\begin{abstract}
Different extensions, such as Transition State theory of Eyring-Polanyi-Evans model of the original Born-Kramers-Slater Model for the Velocity of Chemical Reactions are discussed based on Smoluchowski and Fokker-Plank equations with various properties of Brownian motion and including 1-, 2-, 3-, and multi- dimensional models with applications in Neuroscience.
\end{abstract}

Key words: Fokker-Plank equation, transition state theory, tunneling.

"The rate of chemical reactions is a very complicated subject."

(Harold S. Johnson, 35 years after EPE).

"The overall picture is that the validity of the transition state theory has not yet been really proved and its success seems to be mysterious."

(Raymond Daudel, Georges Leroy, Daniel Peeters, and Michael Sana, 17 years later

in "Variational Transition State Theory with Multidimensional Tunneling" by Antonio Fernandez-Ramos, Benjamin A. Ellingson, Bruce C. Garrett, and Donald G. Truhlar).

\section{Introduction}

There are different questions, arising in the original one-dimensional Kramer's model for application of Brownian motion to kinetics chemical reactions, and consequent extensions to 2-, 3-, and multi-dimensional approaches, concerning state of equilibrium between reactants and products or activated complexes, different filtering patterns, different energy barriers,

Corresponding author: Michael Fundator, master, main research fields: multidimensional time model for probability cumulative function, multi-scale time analysis, stochastic differential equations for reactions kinetics. large depolarization that causes two time scales requiring frequency domain analysis, spatial dependence of memory friction, and other time and probabilistical phenomena that can appear e.g. in application to surface acoustic wave pressure sensors in investigation of the sensitivity of these devices in high pressure and high temperature environments. All of the above mentioned questions require resolution and special and very sensitive approaches from the combination of new mathematical methods, quantum mechanics considerations, and empirical investigations of kinetics of chemical reactions, for 
both modeling and simulation of these parameters.

The discussion with Statement of Chemical Equations, Discussion of Boltzmann Type Kinetic Equations, Kramers Approach with Remaining Problems and Modern Approaches is intended to provide guidance how to deal with arising problems in modeling and simulation.

\section{Materials}

Based on huge number of publications that were stemming from new results and publications in Stochastic Analysis of Differential Equations in relation to chemical kinetics (to mention a few [27-47]), application of Multiphysics and COMSOL, simulating multiple physical models combining chemical kinetics and fluid mechanics with finite element methods for the mathematical structure of the models of Boltzmann type kinetic equations for reacting gas mixtures for particles undergoing inelastic interactions with reactions of bimolecular and dissociation-recombination type is very complicated, because the collisional operators that usually in the full Boltzmann equations, are expressed by 5 -fold integrals. Consequently direct numerical applications of these models present several computational difficulties.

The search for the simpler solution had its long way through the introduction of the equation for the Brownian motion by Albert Einstein and the quantum state tomography (QST) to application of multi-dimensional Fokker-Plank stochastic differential equation. With the application of transition State Theory to Arrhenius equation shown in Eq. (1):

$$
R=C e^{-\left(E_{b} / k T\right)}
$$

where, $R$ is the rate of chemical reaction, $E_{b}$ the activation energy barrier, $k$ is the Boltzmann constant, $T$ is the temperature, and $C$ is a constant transforms $C$ to (Eq. (2)):

$$
C=\frac{k T}{h}
$$

where, $h$ is Plank's constant, this however, does not consider the state of equilibrium of the reactants.

The equilibrium constant is expressed by Eq. (3):

$$
\mathrm{k}=\exp (-\Delta G / R T)
$$

where, $\Delta G$ is Gibbs energy of activation and transition state, and is a difference between energy of reactants and energy of activated complexes shown in Eq. (4):

$$
\mathrm{k}_{1}=\exp (\Delta \mathrm{S} / \mathrm{R}) \exp (-\Delta \mathrm{H} / \mathrm{RT})
$$

where, $\Delta \mathrm{S}$ is an entropy of activation and $\Delta \mathrm{H}$ is an enthalpy of activation Eyring and Polanyi constructed a potential energy surface as a three-dimensional diagram following foundations of quantum-mechanics using experimental data on vibrational frequencies and energies of dissociation.

TST (Transitional State theory) (stated by Eyring, Polanyi and Evans) does not require the reactants and products to be in equilibrium, but the activated complexes are in quasi-equilibrium with the reactants. And the equilibrium constant $\mathrm{k}$ for quasi- equilibrium is Eq. (5):

$$
\mathrm{k}=\frac{k_{1}}{k_{2}} k_{2}=\mathrm{k}^{\prime} v
$$

where, $k_{1}$ is the rate constant, $v$ is the frequency of the vibrational mode converting the activated complex to the product, $k^{\prime}$ is a proportionality constant, and from the statistical mechanics (Eq. (6)):

$$
\mathrm{k}=\frac{k_{b} T}{h v} \exp (-\Delta \mathrm{G} / \mathrm{RT})
$$

with values of interest at the transition state (Eq. (7)).

$$
\Delta \mathrm{G}=\Delta \mathrm{H}-T \Delta \mathrm{S}
$$

where, $\Delta \mathrm{S}$ is an entropy of activation and $\Delta \mathrm{H}$ is an enthalpy of activation and Eyring equation has the following form shown in Eq. (7):

$$
\mathrm{k}=k^{\prime} \frac{k_{b} T}{h} \exp (\Delta \mathrm{S} / \mathrm{R}) \exp (-\Delta \mathrm{H} / \mathrm{RT})
$$

In the theory of the velocity of chemical reactions the problem of study by Kramers was based on assumption that the reactants are in the state of equilibrium, or quasi-equilibrium (chemical equilibrium). His introduction of diffusion equation is 

Motion and Fokker-Plank Equation Including 1D, 2D, 3D, and Multi-dimensional Approaches

given in the following form of Fokker-Plank equation (Eq. (9)):

$$
\mathrm{m} \frac{\partial^{2 x}}{\partial t^{2}}=-\frac{\partial U(x)}{\partial x}-\gamma m \frac{\partial x}{\partial t}+\mathrm{F}(\mathrm{t})
$$

where, $m$ is the reduced mass in the potential of mean force $U$, and $F$ is a noise of a random fluctuating force, originating from the thermal motion, $\gamma$ is a viscosity.

It was based on the assumptions about a particle that moves in an external field of force and additionally is subject to the irregular forces of a surrounding medium in temperature equilibrium, which he called Brownian motion. The conditions are such that the particle is thought of as caught in a potential hole but may escape in the course of time by passing over a potential barrier. The problem is to calculate the probability of escape in its dependency on temperature and viscosity of the medium.

The study had the following problems, pointed out in his original paper:

(1) The study for the sake of simplicity was only a one-dimensional model;

(2) However, as long as no perfect temperature equilibrium is attained, the equation of Max Well velocity distribution holds only approximately. This is even the case when the external force is zero. According to his description, the Brown in forces of the medium illustrate the mechanism which strives to bring about temperature equilibrium. The value of the viscosity coefficient $\gamma$ (which may depend on $T$ even in the manner of an exponential function) is a measure for the intensity with which the molecules in the different states react with the surrounding medium;

(3) The model illustrates also the ambiguity involved in the conception, transition state;

(4) Quantum mechanical, tunnel-effects for which there is no room in our model, could also play a part;

(5) Both Kramers and Grote-Hynes improvement give a well-defined rate constant, and therefore cannot account for dispersed kinetics or dynamic disorder. Such a clear separation of time scale is no longer true for proteins, which are sluggish systems as demonstrated by the fluctuation observed at the slow and broad range of time scales.

Dispersed kinetics and dynamic disorder has been the subject of intensive theoretical investigations. The first approach assumes the fluctuating rate constant is phenomenologically dependent on a time-varying control parameter, such as the activation barrier height, or the area of the bottleneck. Although this approach is conceptually straightforward, the control parameters are usually not experimentally accessible. As a result, their dynamics is often assumed empirically on an $a d$ hoc basis. One of the examples is Brownian motion governed by Langevin dynamics.

The second one assumes a kinetic scheme involving multiple discrete conformational states with different rate constants. However, there is often no sufficient information about the kinetic parameters or the connection topology among the multiple states.

The quantum state tomography QST could be considered as an attempt to solve most of the above problems. Its aim is to statistically reconstruct an unknown state from the outcomes of repeated measurements performed on identical copies of the state. Among the proposed estimation methods the authors mention:

(1) variations of maximum likelihood and least squares estimator;

(2) linear inversion;

(3) Bayesian inference;

(4) estimation with incomplete measurements;

(5) continuous variables tomography.

However, composite systems such as trapped ions, due to the exponential increase in dimension in order to identify and estimate the state with a reduced number of measurements demand special approaches.

RRKM (Rice-Ramsperger-Kassel-Marcus) rate theory developed about the time of BKS (Bohr, Kramers, and Slater) theory that uses notions of active and inactive molecules is a good introduction to the use of well developed mathematical apparatus of 

Motion and Fokker-Plank Equation Including 1D, 2D, 3D, and Multi-dimensional Approaches

Boolean algebra and fuzzy logic. Kramers reaction rate theory predicts that the solvent dynamics will always decrease the reaction rate, or that in the most favorable situation no barrier recrossings take place and the rate constant corresponds with that of RRKM rate theory, which gives the Transition State Theory rate as a function of the collision rate for independent polyatomic molecules. However, for general realistic applications, Kramers' theory (and RRKM theory) fails when the time scale of barrier crossing (which is of course much faster than $1 / \mathrm{k}$ ) is in the same order or even slower than the time scale of the correlations in the random solvent fluctuations.

Both theories BKS and RRKM depend on time scale of barrier escaping and both fail in many particular and experimental cases. In order to obtain a good agreement between experiment and theory, a smaller value seff $<s$ for the total number of the vibrational degrees of freedom should be considered in the classical RRKM theory.

First approach to multidimensional time model is through Kramers turnover problem in the theory of velocity of chemical reactions.

It is important and very interesting to consider such point that Kramers in his original work had it as possibility that multidimensional pattern could be related to time dimensions, as he based his introduction theory of Brownian motion on the Einstein's pattern he considered a range of time intervals $\tau$. His discussion of the possibility of a term proportional to $\tau$ in the expression for Moments of Brownian motion Bin $(\mathrm{n}>1)$ related it to the fact that the values, which $X$ takes at moments $t_{1}, t_{2} \ldots t_{n}$ which lie sufficiently close together are no longer independent; and Moments of Brownian motion B $\tau_{n}$ $(\mathrm{n}>1)$ in fact are represented by a volume integral

$$
\int \ldots \int X\left(\mathrm{t}_{1}\right) X\left(\mathrm{t}_{2}\right) \ldots X\left(\mathrm{t}_{\mathrm{n}}\right) d \mathrm{t}_{1} d \mathrm{t}_{2} \ldots . d \mathrm{t}_{\mathrm{n}}
$$

over an $\mathrm{n}$-dimensional cube; the contribution to this integral due to a narrow cylinder extending along the diagonal $\mathrm{t}_{1}=\mathrm{t}_{2}=\ldots=\mathrm{t}_{\mathrm{n}}$ may give a term proportional to $\tau$.

\section{References}

[1] Fundator, M. 2016. "Applications of Multidimensional Time Model for Probability Cumulative Function for Parameter and Risk Reduction. In JSM Proceedings Health Policy Statistics Section Alexandria." VA: American Statistical Association. 433-41.

[2] Fundator, M. 2016. "Multidimensional Time Model for Probability Cumulative Function." In JSM Proceedings Health Policy Statistics Section. 4029-39.

[3] Fundator, M. 2016. Testing Statistical Hypothesis in Light of Mathematical Aspects in Analysis of Probability doi:10.20944/preprints201607.0069.v1

[4] Fundator, M. 2017. Application of Multidimensional Time Model for Probability Cumulative Function to Brownian Motion on Fractals in Chemical Reactions (44th Middle Atlantic Regional Meeting, June/9-12/16, Riverdale, NY) Academia Journal of Scientific Research (ISSN 2315-7712) DOI: 10.15413/ajsr.2016.0167 In preparation for publication.

[5] Fundator, M. 2017. Application of Multidimensional time model for probability Cumulative Function to Brownian motion on fractals in chemical reactions (Northeast Regional Meeting, Binghamton, NY, October/5-8/16). Academia Journal of Scientific Research (ISSN 2315-7712) DOI: 10.15413/ajsr.2016.0168 In preparation for publication.

[6] Fundator, M. 2017. "Multidimensional Time Model for Probability Cumulative Function and Connections Between Deterministic Computations and Probabilities." Journal of Mathematics and System Science 4: 101-9.

[7] Bohr, N., Kramers, H. A., and Slater, J. C. 1924. "The Quantum Theory of Radiation, Reprinted in Van der Waerden B. L., Sources of Quantum Mechanics (Amsterdam, 1967). The quantum Theory of Radiation Phil. Mag. (6) 47: 785-802.

[8] Hendry, J. 1981. Bohr-Kramers-Slater: A Virtual Theory of Virtual Oscillators and Its Role in the History of Quantum Mechanics. Centaurus, 25: 189-221. doi:10.1111/j.1600-0498.1981.tb00644.x

[9] Eyring, H. 1935. "The Activated Complex in Chemical Reactions." J. Chem. Phys. 3 (2): 107-15. doi:10.1063/1.1749604.

[10] Hänggi, P., Talkner, P., and Borkovec, M. 1990. "Reaction-Rate Theory: Fifty Years After Kramers." Reviews of Modern Physics. 62 (2) 251-342.

[11] Laidler, K., and King, C. 1983. "Development of transition-state theory." Journal Phys. Chem. 87 (15): 2657.

[12] Laidler, K., and King, C. 1998. "A Lifetime of Transition-state Theory." The Chemical Intelligencer 4 (3): 39.

[13] Laidler, K. J. 1969. Theories of Chemical Reaction Rates. Book with editor McGraw-Hill. 

Motion and Fokker-Plank Equation Including 1D, 2D, 3D, and Multi-dimensional Approaches

[14] McCann, L. I., Dykman, M., and Golding, B. 1999. "Thermally Activated Transitions in a Bistable Three-dimensional Optical Trap." Journal Nature 402: 785-7.

[15] Basilevskii, M. V., Ryaboi, V. M., and Weinberg, N. N. 1990. Kinetics of Chemical Reactions in Condensed Media in the Framework of the Two-dimensional Stochastic Model." Journal Phys. Chem. 94 (24): 8734-40.

[16] Davis, M. E., and Davis, R. J. 2003. "Fundamentals of Chemical Reaction Engineering Published." The McGraw-Hill ISBN 0-07-119260-3.

[17] Bianchi, M. P., and Monaco, R. 2004. "A BGK Type Model for a Gas Mixture with Reversible Reactions." in New Trends in Mathematical Physics (World Scientific, Singapore) 107-20.

[18] Kramers, H. A. 1940. "Brownian Motion in a Field of Force and the Diffusion Model of Chemical Reactions." Physical 7 (4) 284-304.

[19] Von Mises, R. 1981. Probability, Statistics and Truth, 2nd rev. Dover Books. New York, ISBN 0-486-24214-5.

[20] Morters, P. 2010. Sample Path Properties of Brownian Motion. Lecture Notes. http://people.bath.ac.uk/maspm/lecnotes.pdf.

[21] Brillinger D. R. 1982. Two Reports On Higher-Order Moments: a) Moments, Cumulants and Some Applications To Stationary Random Processes. b) Distributions of Particle Displacements Via Higher-order Moment Functions. Dig. DB http://digitalassets.lib.berkeley.edu/sdtr/ucb/text/382.pdf.

[22] Tong, Y. L. 1997. "Relationship between Stochastic Inequalities and Some Classical Mathematical Inequalities." Journal of Inequalities and Applications 1 (1): 85-98.

[23] Esary, J. D., Proschan, F., and Walkup, D. W. 1976. "Association of Random Variables with Applications" Journal Ann. Math. Statist. 38: 1466-74.

[24] Halmos, P. R. 1963. "Lectures on Boolean Algebras." Book by Van Nostrand.

[25] Khinchin, A. Y. 1998. "Three Pearls of Number Theory." (Dover Books on Mathematics) ISBN-10: 0486400263

[26] Cramer, M., Plenio, M. B., Flammia, S. T., Somma, R., Gross, D., Bartlett, S. D., Landon-Cardinal, O., Poulin, D., and Liu, Y. K. 2010. "Efficient Quantum State Tomography Nat Commun.” doi: 10.1038/ncomms1147.

[27] Altepeter, J. B., James, D. F. V., and Kwiat P. G. 2004 Quantum State Tomography https://ai2-s2-pdfs.s3.amazonaws.com/cd30/ddbb5a49ef8 12b717ba49b8393615a7140b3.pdf.

[28] Mohseni, M., Rezakhani, A. T., and Lidar, D. A. 2008. Quantum Process Tomography: Resource Analysis of Different Strategies arXiv:quant-ph/0702131.

[29] Acharya, A., Theodore, K. T., and Guță, M. 2016. "Statistically Efficient Tomography of Low Rank States with Incomplete Measurements.” New Journal of Physics 18 (4): 043018.

[30] Wikipedia.

[31] Rjasanow, S., and Wagner, W. 2005. Stochatic Numerics for the Boltzmann Equation Book by Springer ISBN 978-3-540-27689-0.

[32] Donald, L. T. 1998. "Modern Methods for Multidimensional Dynamics Computations in Chemistry." Book by World Scientific ISBN-10: 9810233426.

[33] Atkins, P., and de Paua, J. 2006. "Physical Chemistry for the Life Sciences." Books by Oxford University Press. 2006. ISBN-10: 1-4292-3114-9.

[34] Garrett, R., and Grisham, C. 2005. Biochemistry. 3rd Edition. Book by California. Thomson Learning, Inc.

[35] Wade, L. G. 2006. Organic Chemistry. 6th Edition. Book by Pearson Prentice Hall.

[36] The Arrhenius Law: Activation Energies-Chemistry LibreTexts https://chem.libretexts.org > ... > Kinetics > Temperature Dependence of Reaction Rates.

[37] Chemical Reactions and Kinetics http://chemed.chem.purdue.edu/genchem/topicreview/bp/ ch22/react.html.

[38] Griffiths, J. 2008. "A Few of the Great People and Major Discoveries that Have Shaped this Century-old Technique." Journal Anal. Chem. 80 (15): 5678-83.

[39] Hänggi, P., and Goychuk, I. 2002. "Ion Channel Gating: A First-passage Time Analysis of the Kramers Type." PNAS 99 (6): 3552-6.

[40] Das, S., Vikalo, H., and Hassibi, A. 2009. "Stochastic Modeling of Reaction Kinetics in Biosensors Using the Fokker Planck Equation." IEEE International Workshop on Genomic Signal Processing and Statistics, Minneapolis, MN. 1-4.

[41] Lecca, P. 2013. "Stochastic Chemical Kinetics: A Review of the Modelling and Simulation Approaches." Biophysical Reviews 5 (4): 323-45.

[42] Oppenheim, I., Shuler, K. E., and Weiss, G. H. 1969. "Stochastic and Deterministic Formulation of Chemical Rate Equations." Journal of Chemical Physics 50 (1): 460-6.

[43] Moore, C. B. A. "Spectroscopist's View of Energy States." Energy Transfers, and Chemical Reactions.

[44] Reinhardt, C. 2006. "Shifting and Rearranging: Physical Methods and the Transformation of Modern Chemistry." Nuncius Journal of the History of Science 55 (1): 93-4.

[45] Daniel T. Stochastic Simulation of Chemical Kinetics。

[46] Scheraga, H. A., Khalili, M., and Liwo, A. Protein-Folding Dynamics: Overview of Molecular Simulation Techniques

[47] Aristov V.V. 2001. "Direct Methods for Solving the Boltzmann Equation and Study of Nonequilibrium Flows." Springer Netherlands. 60. 